\title{
Aprendiendo a desarrollar sistemas de evaluación formativa y compartida en las prácticas como maestra de Educación Física
}

\section{Learning to develop systems of formative and shared assessment in the practices as a teacher of Physical Education in Primary School}

\author{
Carla Fernández Garcimartín, Víctor M. López Pastor² y Cristina Pascual \\ Arias $^{3}$
}

Fecha de recepción: 19/01/2019; Fecha de revisión: 25/02/2019; Fecha de aceptación: 25/03/2019

Cómo citar este artículo:

Fernández-Garcimartín, C., López-Pastor, V. M., \& Pascual-Arias, C. (2018). Aprendiendo a desarrollar sistemas de evaluación formativa y compartida en las prácticas como maestra de Educación Física en Primaria. Revista de Innovación y Buenas Prácticas Docentes, 8, 119-131.

Autor de Correspondencia: Carla Fernández Garcimartín, carlafdzg96@gmail.com

\begin{abstract}
Resumen:
En este trabajo mostramos una experiencia sobre la utilización de un sistema de Evaluación Formativa y Compartida $(\mathrm{EFyC})$ en un aula de primaria en el área de educación física, durante el periodo de prácticas docentes, y ligado a un proyecto de innovación docente sobre la trasferencia universidad-escuela de estos modelos de evaluación. Se ha realizado una UD completa con $5^{\circ}$ de Primaria con este sistema de evaluación utilizando los siguientes instrumentos: diario del profesor, cuaderno del alumno, fichas de observación grupales, fichas de seguimiento individual, cuestionarios de autoevaluación, fichas de autocalificación, fichas de coevaluación, escala graduada; y distintas técnicas. Los resultados nos muestran que este sistema de EFyC ha permitido al alumnado tener un conocimiento real y continuo de su aprendizaje en Educación Física gracias al feedback continuo de la maestra. La aplicación de este sistema ha favorecido un clima de confianza entre los alumnos y a la maestra le ha permitido una cercanía y un seguimiento más individual y positivo de ellos.

Palabras clave: Evaluación Formativa, Evaluación Compartida, Educación Física, Educación Primaria.
\end{abstract}

\section{Abstract:}

In this paper, we show a real experience of the formative and shared assessment in a class of primary school in physical education, during the period of teaching practices, and linked to a teaching innovation project about the university-school transfer of this assessment model. The results found will also be analysed. We have designed a unit of work for a 5th grade primary class using the following instruments: a teacher's diary, student's notebooks, group observation records, individual follow-up sheets, selfassessment questionnaires, self-qualification cards, co-assessment cards and a graded scale; and the following techniques. Results show us that this system of formative and shared assessment has allowed the students to have a real and continuous knowledge of their learning in Physical Education thanks to the continuous feedback of the teacher. The application of this system has favored a climate of trust between the students and the teacher that has allowed a closer and more individual and positive follow-up of them. Key Words: Formative Assessment, Shared Assessment, Physical Education, Primary School.

\footnotetext{
1 Universidad de Valladolid (España), carlafdzg96@gmail.com

2 Universidad de Valladolid (España), vlopez@mpc.uva.es

3 Universidad de Valladolid (España), cristinapascuari@gmail.com
} 


\section{INTRODUCCIÓN}

En este artículo presentamos una experiencia de Evaluación Formativa y Compartida (EFyC) desarrollada en un aula de $5^{\circ}$ curso de educación primaria. Lo que tiene de especial esta experiencia es que ha sido llevada a cabo durante el prácticum de $4^{\circ}$ curso del grado de Maestro de Primaria, mención de Educación Física. También está ligada a un Proyecto oficial de Innovación Docente que se desarrolla en la Facultad de Educación de Segovia (Universidad de Valladolid) sobre la trasferencia universidadescuela de estos modelos de evaluación. A continuación explicaremos el contexto educativo donde se lleva a cabo. En los siguientes apartados se explicará el sistema de evaluación utilizado, los principales resultados conseguidos y las conclusiones.

\subsection{Contexto educativo}

La experiencia se lleva a cabo en un centro educativo de la parte moderna de la ciudad de Segovia. Es un centro pequeño y familiar de una línea, que tiene solamente 210 alumnos. Las instalaciones del centro son muy completas y dispone de gimnasio cubierto, dos patios amplios y disponibilidad de uso de un pabellón polideportivo municipal muy cercano.

El grupo con el que se lleva a cabo la experiencia es el de $5^{\circ}$ de Primaria, que cuenta con 24 alumnos, o de los cuales son ACNEE (Alumnos Con Necesidades Educativas Especiales). Aunque es un grupo muy heterogéneo, todos siguen un ritmo normal de clase, sin que eso afecte al aprendizaje. La experiencia se lleva a cabo en la asignatura de educación física (EF), que tiene asignadas dos horas y media semanales.

\subsection{Unidad didáctica en que se lleva a cabo la experiencia}

La experiencia de EFyC se lleva a cabo en la unidad didáctica (UD) titulada: "Me importa mi salud", que está relacionada con el aprendizaje de los tipos de salud, la adquisición de hábitos saludables y la corrección de malos hábitos y mitos que se dan sobre la salud. Los objetivos principales a trabajar en esta UD son:

1. Conocer los diferentes tipos de salud de forma práctica a través del descubrimiento guiado.

2. Formar parte del proceso de aprendizaje personal de la salud.

3. Reconocer los errores que cometemos día a día que afectan a nuestra salud.

4. Llevar a cabo hábitos saludables de forma correcta en nuestro día a día.

5. Desarrollar un sentido crítico en relación a nuestra salud personal y a la de los demás.

Las principales actividades de aprendizaje, a trabajar a lo largo de toda la UD, son las siguientes:

1. Cuaderno del alumno: en él apuntan lo que se ha trabajado en la sesión, cómo se ha llevado a cabo, lo que se ha aprendido (como breve reflexión personal) y un dibujo libre sobre lo trabajado. Asentamos lo aprendido durante la sesión.

2. Explicaciones previas, donde el profesor muestra el contenido a trabajar y se lanzan preguntas para que los propios alumnos sepan qué se va a trabajar y lo más importante de lo que se va a realizas. Por ejemplo: iniciamos el atletismo, el profesor pregunta qué tipos de pruebas hay y con las respuestas de los alumnos corregimos errores y llegamos a la respuesta correcta.

3. Calentamiento: al comienzo de cada sesión realizamos movilidad articular y estiramientos explicando los beneficios del calentamiento y las especificaciones de cada ejercicio (cómo hacerlo bien y mal, qué parte del cuerpo estamos trabajando, por qué hacemos hincapié en unas zonas y no en otras, etc.).

4. Actividades de aprendizaje vivenciado, en la parte central y mayoritaria de cada sesión, según aparecen reflejadas en la figura 1, organizada por sesiones. 


\begin{tabular}{|c|c|c|}
\hline Sesión & Actividad & Explicación \\
\hline 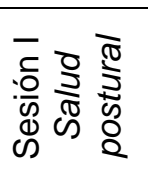 & $\begin{array}{l}\text { "Enseño a } \\
\text { mi cuerpo" }\end{array}$ & $\begin{array}{l}\text { Representación motriz (no verbal, expresión corporal) de dos } \\
\text { situaciones cotidianas en las que se muestre cómo se realiza una } \\
\text { postura correctamente y otra cómo se hace normalmente dañando al } \\
\text { cuerpo }\end{array}$ \\
\hline 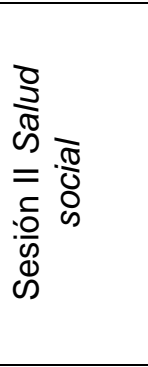 & $\begin{array}{l}\text { "Recojo } \\
\text { agua, } \\
\text { como } \\
\text { todos los } \\
\text { días" }\end{array}$ & $\begin{array}{l}\text { Coordinación y cooperación. Se divide a la clase en dos grupos: unos } \\
\text { serán los que vayan a recoger agua, y otros los que esperen en la } \\
\text { aldea. Cuando estos hayan ido a por agua, se cambiarán los roles, } \\
\text { pero esta vez, podrán tener ayuda de un compañero que les guíe. El } \\
\text { espacio estará lleno de obstáculos y los barreños pesarán mucho, } \\
\text { además en la primera ronda, algunos irán con una mano solamente y } \\
\text { otros no verán nada. En la segunda estarán los barreños del grupo } 1 \\
\text { a un lado por si quieren ayudar y repartirse el peso entre la pareja, } \\
\text { seguirán con dificultades pero el compañero los podrá ayudar y guiar. }\end{array}$ \\
\hline \multirow{2}{*}{ 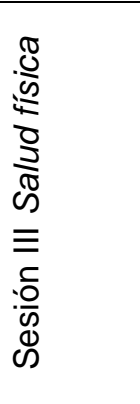 } & $\begin{array}{l}\text { "Tres en } \\
\text { raya } \\
\text { humano" }\end{array}$ & $\begin{array}{l}\text { Trabajamos a partir de las tres en raya para conocer los mitos físicos } \\
\text { que se dan en nuestros días. El equipo que gane se lleva un mito que } \\
\text { compartirá con los demás al finalizar el juego. }\end{array}$ \\
\hline & $\begin{array}{l}\text { "Circuito } \\
\text { físico } \\
\text { injusto" }\end{array}$ & $\begin{array}{l}\text { Circuito formado por ejercicios básicos (A) y ejercicios más fuertes } \\
\text { (B). Por parejas, los que tengan la letra (A) comenzarán por esos } \\
\text { ejercicios y acabarán por la letra (B), después se tomarán las } \\
\text { pulsaciones nada más acabar (lo anota su pareja). La pareja (B) } \\
\text { comenzará por los ejercicios de la letra (B) y terminará por los (A), se } \\
\text { toma pulsaciones y lo anota su pareja. Se compara el esfuerzo y las } \\
\text { pulsaciones empezando por ejercicios suaves o más fuertes. }\end{array}$ \\
\hline \multirow{3}{*}{ 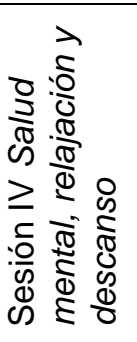 } & $\begin{array}{l}\text { "Rincón de } \\
\text { los } \\
\text { contrastes" }\end{array}$ & $\begin{array}{l}\text { Técnica de Jacobson para contraer y relajar los músculos trabajando } \\
\text { la concentración en esas partes del cuerpo. }\end{array}$ \\
\hline & $\begin{array}{l}\text { "Rincón de } \\
\text { pilates " }\end{array}$ & $\begin{array}{l}\text { Iniciación al pilates con tres ejercicios básicos de estiramientos, } \\
\text { trabajo de la zona abdominal y de espalda que pueden llevar a casa } \\
\text { como rutina diaria. }\end{array}$ \\
\hline & $\begin{array}{l}\text { "Rincón del } \\
\text { spa" }\end{array}$ & $\begin{array}{l}\text { Masajes por parejas según lo que va diciendo la profesora explicando } \\
\text { qué hacemos y por qué se hace de esa manera }\end{array}$ \\
\hline \multirow{3}{*}{ 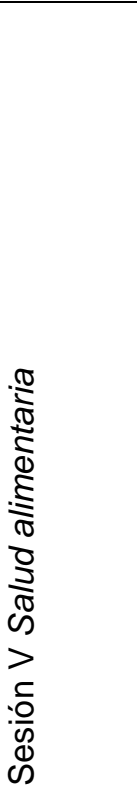 } & $\begin{array}{l}\text { "Rincón del } \\
\text { gluten" }\end{array}$ & $\begin{array}{l}\text { Trabajaremos con diferentes tipos de alimentos que contengan gluten } \\
\text { y aquellos que no lo contienen. El grupo se dividirá en pacientes y } \\
\text { médicos, entonces los alumnos pacientes en una maqueta del } \\
\text { aparato digestivo deberán introducir aquellos alimentos que no } \\
\text { contienen gluten, para que después, los médicos, diseccionen el } \\
\text { estómago y el intestino delgado para comprobar si los pacientes han } \\
\text { ingerido los alimentos adecuados a su alergia }\end{array}$ \\
\hline & $\begin{array}{l}\text { "Rincón de } \\
\text { los lácteos" }\end{array}$ & $\begin{array}{l}\text { Llevamos etiquetas de productos alimentarios que hay en todas las } \\
\text { cocinas quitando la parte de ingredientes. Los alumnos deberán } \\
\text { señalar si estos contienen lácteos o no. Sin decirles la respuesta } \\
\text { correcta, les daremos, ahora sí, la parte de los ingredientes y deberán } \\
\text { relacionar cada ingrediente con su producto. Se darán cuenta de que } \\
\text { muchos productos que al parecer no tienen los nutrientes que } \\
\text { componen los lácteos, sí que los tienen y puede ser peligroso para } \\
\text { cualquier persona. Fomentaremos la visualización crítica de los } \\
\text { ingredientes de los productos }\end{array}$ \\
\hline & $\begin{array}{l}\text { "Relevos } \\
\text { saludables" }\end{array}$ & $\begin{array}{l}\text { Relevos de atletismo trabajados anteriormente. Cambiaremos el } \\
\text { testigo por un tupper y, en cada puesto, deberán rellenarlo con un } \\
\text { trozo de fruta para completar la carrera y comerse lo que hayan } \\
\text { conseguido }\end{array}$ \\
\hline
\end{tabular}

Figura 1. Actividades de aprendizaje vivenciado. 


\begin{tabular}{|c|c|c|}
\hline Sesión & Actividad & Explicación \\
\hline 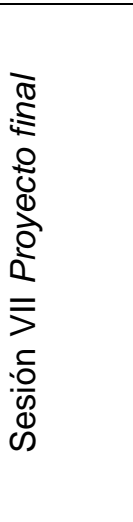 & $\begin{array}{l}\text { "Me importa } \\
\text { mi salud" }\end{array}$ & $\begin{array}{l}\text { Trabajo por grupos de la pirámide NAOS. Se hace una reflexión } \\
\text { sobre todas las pirámides y se corrigen ideas (voy pasando por } \\
\text { los grupos). En los mismos grupos, repasamos los tipos de salud } \\
\text { trabajados estos días y, cada uno, elige uno. Deberán preparar } \\
\text { una explicación como si fueran algún famoso, de la tele, } \\
\text { periodista, madre, padre, profesor, etc. y una propuesta de cómo } \\
\text { se lleva a cabo esa propuesta saludable en nuestro día a día. } \\
\text { (Que sean capaces de transmitir lo que han aprendido, de la } \\
\text { importancia que tiene y que lo disfruten. El tema será libre de } \\
\text { todo lo trabajado (alimentos, posturas...); cada grupo de una } \\
\text { cosa.) } \\
\text { Enseñarlo a los alumnos de } 1^{\circ} \text { EP y ellos coevalúan a los de } 5^{\circ} \\
\text { EP. }\end{array}$ \\
\hline
\end{tabular}

Figura 1. Actividades de aprendizaje vivenciado (continuación).

Fuente: Elaboración propia.

\section{DESARROLLO DE LA EXPERIENCIA DE INNOVACIÓN}

\subsection{Explicación general del sistema de EFyC}

El sistema de EFyC que se va a poner en práctica parte de una serie de instrumentos y técnicas de evaluación para evaluar de forma completa y coherente. Este sistema está basado en el diálogo y el intercambio de ideas para mejorar los procesos de enseñanza y aprendizaje de los alumnos, y del maestro que lo desarrolla (LópezPastor, 2006). A continuación, se muestra cómo se va a desarrollar este sistema de evaluación.

1. Los alumnos reciben la información sobre cómo van a participar en su evaluación y qué van a tener que hacer. Para ello se les da el primer día la rúbrica de aprendizaje (figura 3) con todos los criterios a evaluar. Se explica y, al final de la UD, se autoevalúan rellenándola. El maestro hace lo mismo, pero evaluando a cada alumno con la rúbrica de aprendizaje.

2. Como aparece en la figura 1 , en la última sesión los alumnos de $1^{\circ}$ de primaria evaluarán a los alumnos de $5^{\circ}$ desarrollándose así una evaluación triangulada desde otro punto de vista (coevaluación figura 5). Los alumnos se autoevalúan al final de cada sesión (figura 4). La profesora compara los resultados de cada autoevaluación con sus anotaciones en las FSG (figura 7), diario del profesor y fichas de coevaluación (figura 5).

3. Después de cada sesión, los alumnos anotan en su cuaderno: qué se ha trabajado, cómo se ha llevado a cabo y qué han aprendido (como reflexión personal breve). Este cuaderno lo corrige la profesora semanalmente y se devuelve con correcciones. Este feedback ayuda al alumnado a saber cómo va trabajando y qué tiene que mejorar para la semana siguiente, cuando la profesora se vuelve a llevar los cuadernos.

4. Se lleva a cabo la evaluación compartida. En una entrevista se comparan primero las rúbricas ya rellenas y se justifican. La profesora muestra los datos evidentes en los que se ha basado para completarla (FSG, diario del profesor y fichas de coevaluación) y se discuten los resultados.

En la figura 2 se muestran esquemáticamente los instrumentos que se utilizan en la experiencia. 


\begin{tabular}{|c|c|c|}
\hline $\begin{array}{l}\text { Técnicas de } \\
\text { evaluación }\end{array}$ & $\begin{array}{l}\text { Instrumentos de } \\
\text { evaluación }\end{array}$ & Puesta en práctica \\
\hline \multirow[t]{2}{*}{$\begin{array}{l}\text { Observación } \\
\text { participante }\end{array}$} & $\begin{array}{l}\text { Diario del } \\
\text { profesor }\end{array}$ & $\begin{array}{l}\text { Cuando lo necesito lo uso como "anecdotario. Anoto los aspectos } \\
\text { más significativos en su diario de clase tras la sesión. Todas las } \\
\text { anotaciones sirven para llevar una evaluación más individualizada y } \\
\text { completa de cada alumno. Así hacemos evidente y real la } \\
\text { evaluación. El diario se utiliza también como herramienta de } \\
\text { reflexión docente. }\end{array}$ \\
\hline & $\begin{array}{l}\text { Ficha de } \\
\text { seguimiento } \\
\text { grupal (FSG) } \\
\text { (tabla 10), e } \\
\text { individual (FSI) } \\
\text { (tabla 9) }\end{array}$ & $\begin{array}{l}\text { En cada sesión se observa a un grupo de } 12 \text { alumnos y se va } \\
\text { rotando cada día el grupo de alumnos. Dado que la UD dura } 6 \\
\text { sesiones, cada alumno será observado durante tres días, al mismo } \\
\text { tiempo que se organiza la clase y se interviene dando feedback. } \\
\text { Las valoraciones se suelen realizar al finalizar la clase, cuando se } \\
\text { tiene un rato para ello; porque durante la clase se está centrado en } \\
\text { las intervenciones docentes y en dar feedback. }\end{array}$ \\
\hline $\begin{array}{l}\text { Revisión } \\
\text { cuaderno del } \\
\text { alumno }\end{array}$ & $\begin{array}{l}\text { Cuaderno del } \\
\text { alumno }\end{array}$ & $\begin{array}{l}\text { Los alumnos deberán realizar su cuaderno de clase semanalmente. } \\
\text { En él incorporarán: (1) qué se ha trabajado, (2) qué han aprendido } \\
\text { y (3) una reflexión personal abierta (además, si quieren, hacen } \\
\text { dibujo libre). Se corrige semanalmente recogiéndolo los lunes o los } \\
\text { viernes y devolviéndolo dos días después de haberlo entregado con } \\
\text { el feedback suficiente para mejorar el cuaderno semanalmente. } \\
\text { Todo este seguimiento de cada alumno será anotado en la FSG. }\end{array}$ \\
\hline $\begin{array}{l}\text { Evaluación } \\
\text { entre iguales }\end{array}$ & $\begin{array}{l}\text { Ficha de } \\
\text { evaluación entre } \\
\text { iguales (tabla 5) }\end{array}$ & $\begin{array}{l}\text { Evaluación entre iguales que se da de forma individual en la sesión } \\
6 \text { (proyecto final). Participan alumnos externos a las sesiones } \\
\text { trabajadas (los de } 1^{\circ} \text { EP co-evalúan a los de } 5^{\circ} \mathrm{EP} \text { ) para mostrar } \\
\text { una evaluación triangulada con otros puntos de vista, de cara al } \\
\text { cierre de la UD. Se da el feedback oral al compañero y escrito en la } \\
\text { misma ficha. }\end{array}$ \\
\hline $\begin{array}{l}\text { La reflexión } \\
\text { docente }\end{array}$ & $\begin{array}{l}\text { Diario del } \\
\text { profesor }\end{array}$ & $\begin{array}{l}\text { Durante cada sesión se anotan los sucesos más relevantes en el } \\
\text { cuaderno del profesor (hora-suceso-alumno). Servirá como una } \\
\text { prueba más de evaluación del aprendizaje de los alumnos e incluso } \\
\text { del maestro. Tras cada sesión reflexiono sobre lo que ha pasado, } \\
\text { así me sirve como autoevaluación y mejora para la siguiente } \\
\text { sesión. Este diario se usa también como anecdotario de situaciones } \\
\text { concretas e importantes que pasan durante la sesión. Las } \\
\text { reflexiones semanales sobre cada día de clase también las anoto } \\
\text { en el diario del profesor, aunque las desarrollo más en un } \\
\text { documento a ordenador. }\end{array}$ \\
\hline $\begin{array}{l}\text { Evaluación } \\
\text { compartida }\end{array}$ & $\begin{array}{l}\text { Rúbrica de } \\
\text { aprendizaje } \\
\text { (tabla 3) }\end{array}$ & $\begin{array}{l}\text { Al comienzo de la UD se lee conjuntamente la rúbrica y se explican } \\
\text { todos los apartados. La pegarán en su cuaderno para revisarla } \\
\text { siempre que quieran. Habrá una pegada en la pared donde } \\
\text { normalmente damos educación física. Al final de la UD: (1) Cada } \\
\text { alumno se autoevalúa y se ubica según donde piensa que se } \\
\text { encuentra en cada criterio; (2) El profesor hace lo mismo con cada } \\
\text { alumno para comparar conjuntamente. Todo este proceso se lleva } \\
\text { a cabo a partir de diálogos individuales entre el profesor y el } \\
\text { alumno mostrando los resultados de todos los instrumentos de } \\
\text { evaluación utilizados durante la UD. Se intercambia información en } \\
\text { torno a los procesos de enseñanza-aprendizaje, autoevaluaciones, } \\
\text { coevaluación, observación, feedback para mejorar, correcciones, } \\
\text { etc. Se concluye llegando a un punto común dentro del salto a la } \\
\text { calificación. }\end{array}$ \\
\hline
\end{tabular}

Figura 2. Instrumentos y técnicas de evaluación utilizados.

Fuente: Adaptado de López-Pastor y Pérez-Pueyo (2017). 


\begin{tabular}{|c|c|c|}
\hline $\begin{array}{l}\text { Entrevistas } \\
\text { individuales } \\
\text { y calificación } \\
\text { dialogada }\end{array}$ & $\begin{array}{l}\text {-Escala } \\
\text { graduada para } \\
\text { autoevaluación y } \\
\text { autocalificación } \\
\text { alumnado } \\
\text {-Cuaderno de los } \\
\text { alumnos } \\
\text {-Diario del } \\
\text { profesor } \\
\text {-Lista de clase } \\
\text {-FSG }\end{array}$ & $\begin{array}{l}\text { Último día de la UD: normalmente los lunes (30 minutos) hablaré } \\
\text { con todos en grupos de } 4 \text { ó } 5 \text { alumnos. (1) Llamo al primer alumno } \\
\text { de la lista; (2) me muestra su rúbrica rellena según su evaluación y } \\
\text { calificación personal; (3) le muestro mi rúbrica de su evaluación y } \\
\text { calificación; (4) comparamos resultados; (5) diálogo tanto si } \\
\text { coinciden los resultados como si no; (5) le muestro los instrumentos } \\
\text { de evaluación utilizados para evaluarle personalmente, datos } \\
\text { concretos del diario del profesor, autoevaluaciones, etc.; (6) } \\
\text { mientras estoy dialogando con el primer alumno, el segundo de la } \\
\text { lista se aparta y va completando su rúbrica para llegar a una } \\
\text { calificación común conmigo. Los resultados de estas entrevistas } \\
\text { individuales se reflejarán en la FSG en el apartado de cada alumno. }\end{array}$ \\
\hline
\end{tabular}

Figura 2. Instrumentos y técnicas de evaluación utilizados (continuación).

Fuente: Adaptado de López-Pastor y Pérez-Pueyo (2017).

\subsection{Ejemplos de algunos de los instrumentos de evaluación utilizados}

En este apartado vamos a presentar ejemplos de los diferentes instrumentos de evaluación utilizados.

\subsection{Sistema de calificación}

El sistema de calificación que se va a poner en práctica consta de dos partes:

1. El sistema de calificación está ajustado a los criterios establecidos en la escala descriptiva (rúbrica) (figura 3), que se presenta y explica desde el primer momento al alumnado. Al finalizar la UD tendrán que rellenar la tabla 4 según lo que ellos consideren de su evolución y aprendizaje en la UD (autoevaluación). El profesor hará lo mismo sobre el trabajo de cada alumno basándose en los diferentes instrumentos de evaluación, dando una información coherente y objetiva del trabajo individual de cada alumno. Es importante aclarar que la figura 3 se presenta a los alumnos con una escala verbal, para que no la relacionen con una calificación numérica, y así intentar ir reduciendo la obsesión por la nota y ayudando a que se centren en el aprendizaje. Para finalizar con la parte de evaluación compartida se realizarán las entrevistas con cada alumno. Cada alumno completa su escala descriptiva (figura 3) y también el profesor, con todos los datos recogidos de cada alumno. Tras el diálogo y el consenso sobre la evaluación a partir de esa escala descriptiva, se pasan los resultados de la evaluación compartida a una última escala graduada para la autocalificación alumnado (tabla 1), pasando esos resultados verbales en numéricos de forma que demos el salto a la "calificación dialogada". La nota final que se ponga es un número que refleja todo el proceso de aprendizaje de cada alumno. Lo importante es que los alumnos se quedan con el proceso de aprendizaje realizado gracias a su trabajo constante y compartido con el profesor. De esta forma sabrán qué hay detrás de la calificación final.

En la tabla 1 se presentan los criterios de evaluación y la conversión de escala verbal a numérica para hacer más fácil el salto a la calificación. 
Tabla 1.

Escala graduada para autoevaluación y autocalificación alumnado.

\begin{tabular}{|c|c|}
\hline $\begin{array}{l}\text { Aspectos a valorar. } \\
\text { Redondea con el bolígrafo las frases que mejor describan tu trabajo y logros en } \\
\text { esta unidad didáctica. }\end{array}$ & Grado \\
\hline $\begin{array}{l}\text {-Siempre participo y me ofrezco para realizar lo que manden y ayudo a mis compañeros } \\
\text { sin que me lo diga el profesor. } \\
\text {-Sé reconocer los errores que cometemos en todos los tipos de salud y soy capaz de } \\
\text { corregirlos en mi día a día. } \\
\text {-Propongo ideas sobre salud que aplico en mi rutina. Se lo explico a mis compañeros. } \\
\text {-Siempre cumplo las normas sin que el profesor me llame la atención. Corrijo a mis } \\
\text { compañeros cuando es necesario. } \\
\text {-Al terminar cada clase hago el cuaderno como me ha dicho el profesor y de manera } \\
\text { ordenada, limpia y reflexiva. } \\
\text {-Siempre escucho al profesor sin que me llame la atención. }\end{array}$ & $\begin{array}{l}\text { MUY } \\
\text { ALTO } \\
(9-10)\end{array}$ \\
\hline $\begin{array}{l}\text {-Normalmente participo en las sesiones y, a veces, ayudo a mis compañeros. } \\
\text {-Sé reconocer los errores que cometemos en cada tipo de salud que trabajemos y estoy } \\
\text { intentando corregirlos. } \\
\text {-Propongo algunas ideas sobre el tipo de salud a trabajar. Si me lo piden mis } \\
\text { compañeros, se lo explico. } \\
\text {-Cumplo las normas sin que me lo diga el profesor. } \\
\text {-A veces hago el cuaderno según acaba la clase. A veces sigo las pautas que me ha } \\
\text { mandado el profesor: ordenado, limpio y reflexivo. } \\
\text {-Escucho al profesor, aunque a veces me llame la atención. }\end{array}$ & $\begin{array}{l}\text { ALTO } \\
(7-8)\end{array}$ \\
\hline $\begin{array}{l}\text {-Me cuesta participar en las sesiones y no suelo colaborar con mis compañeros. } \\
\text {-Me cuesta reconocer los errores que se cometen en cuanto a la salud y no los corrijo } \\
\text { en mi día a día. } \\
\text {-Me cuesta proponer ideas sobre la salud en nuestra vida si el profesor no me pide que } \\
\text { lo haga. } \\
\text {-Me cuesta cumplir las normas y, por eso, el profesor me llama muchas veces la } \\
\text { atención. } \\
\text {-Me cuesta seguir el ritmo de trabajo del cuaderno. A veces lo entrego tarde y/o muy } \\
\text { poco elaborado. } \\
\text {-No suelo escuchar al profesor, me llama la atención. }\end{array}$ & $\begin{array}{l}\text { BAJO } \\
(5-6)\end{array}$ \\
\hline $\begin{array}{l}\text {-No reconozco los errores que se cometen en cuanto a la salud y no los corrijo en mi } \\
\text { día a día. } \\
\text {-No propongo ideas sobre la salud en nuestro día a día, aunque me lo pida el profesor. } \\
\text {-No cumplo las normas. Constantemente el profesor me llama la atención. } \\
\text {-No entrego el cuaderno el día marcado y/o no me esfuerzo en hacerlo correctamente. } \\
\text {-No escucho al profesor, me llama la atención constantemente. }\end{array}$ & $\begin{array}{l}\text { MUY } \\
\text { BAJO } \\
(1-4)\end{array}$ \\
\hline
\end{tabular}

\subsection{Resumen de actividades aprendizaje - evaluación formativa a realizar y salto a la calificación.}

En la figura 3 presentamos un resumen de las relaciones que existen entre las actividades de aprendizaje que se realizan, el sistema de evaluación formativa que se utiliza en dichas actividades y los criterios utilizados para dar el salto a la calificación al final de la unidad didáctica. Se trata de una tabla que ayuda a comprobar el nivel de coherencia curricular o alineación curricular que tiene la UD. 


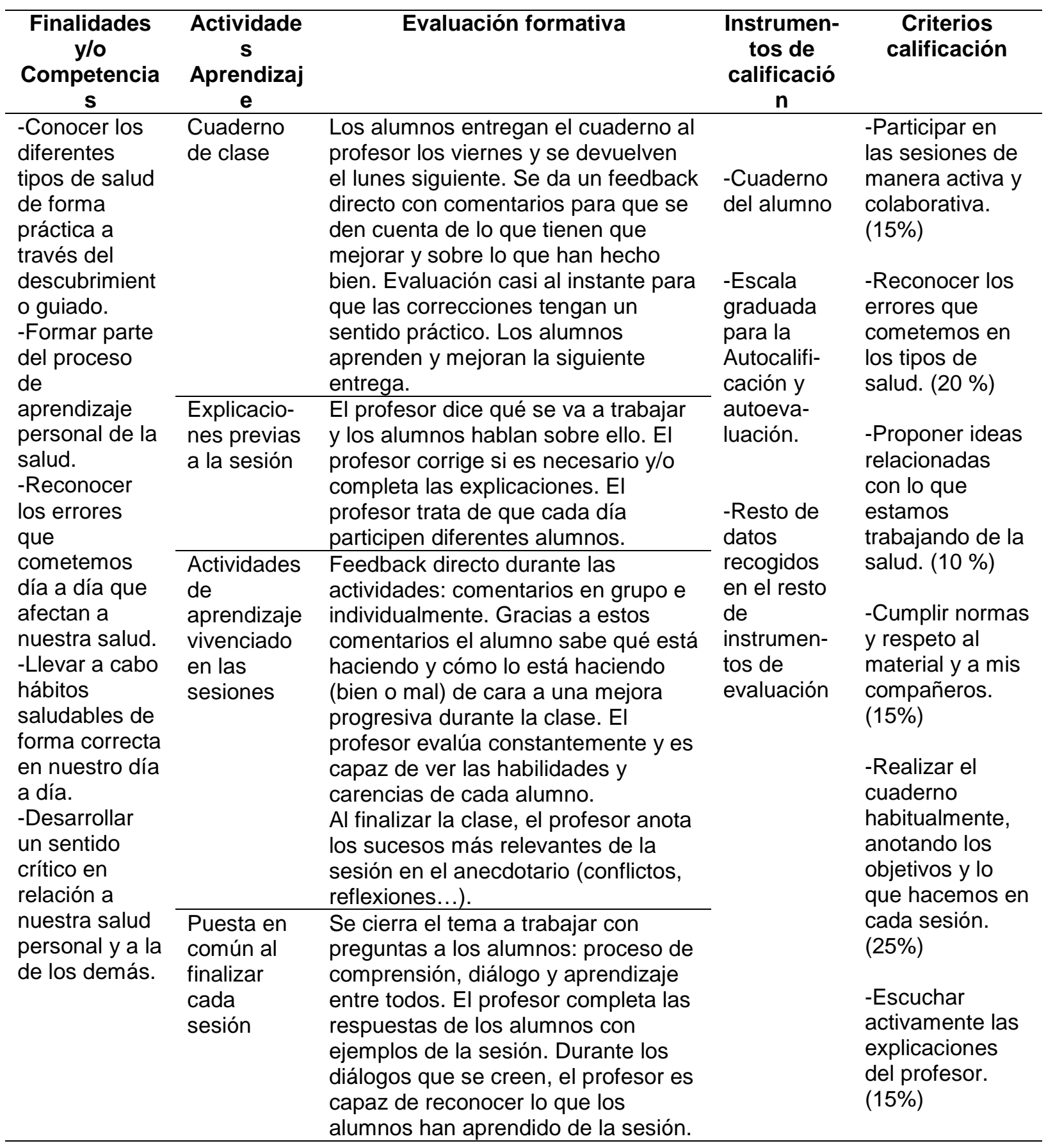

Figura 3. Alineación curricular y nivel de coherencia interna entre los diferentes elementos curriculares (elaborado a partir del informe estructurado de la Red de evaluación formativa en educación para el curso 2017-18).

\section{RESULTADOS}

\subsection{Resultados en el rendimiento académico}

En la tabla 2 mostramos los resultados académicos tras la puesta en práctica de la UD con $5^{\circ}$ de Primaria, organizados por porcentajes y por número de alumnos. 
Tabla 2

Resultados académicos de la UD realizada.

\begin{tabular}{ccc}
\hline & Resultados globales & \\
\hline Calificación & Porcentaje & $\mathbf{N}^{\circ}$ alumnos/as \\
\hline Sobresaliente (9-10) & $20,83 \%$ & 5 \\
\hline Notable (7-8) & $62,5 \%$ & 15 \\
\hline Aprobado (5-6) & $16,66 \%$ & 4 \\
\hline Suspenso (1-4) & $0 \%$ & 0 \\
\hline Totales & $\mathbf{1 0 0} \%$ & $\mathbf{2 4}$
\end{tabular}

Los resultados en el rendimiento académico del alumnado han sido muy positivos, tanto cuantitativamente como cualitativamente. Hay que ser conscientes de la dificultad que tiene convertir todo el proceso de aprendizaje realizado a una única calificación numérica y que ésta sea lo más justa posible. En este sentido, la utilización de la escala graduada ha demostrado ser un instrumento muy útil. Realizar de forma encadenada el proceso de autocalificación y de calificación dialogada nos ha permitido comprobar que cada alumno ha sido consciente del aprendizaje y el trabajo personal realizado durante la UD llevada a cabo, y es capaz de demostrarlo en la entrevista final utilizando las fichas de autoevaluación y su cuaderno.

Como vemos en la tabla 2, ningún alumno ha suspendido esta UD, debido a su implicación y trabajo continuo, lo cual ha servido para generar suficiente aprendizaje para cumplir con los criterios de calificación marcados. En el proceso de autocalificación, un alumno se puso como nota un 4, siendo consciente de que había faltado a tres de las seis sesiones de la UD y no había entregado el cuaderno. Tras la entrevista y la calificación dialogada, este alumno aprobó con un 5 debido a los criterios acordados en la escala descriptiva sobre cómo había trabajado esas tres sesiones.

Las calificaciones han sido muy altas y justas (habiendo coincidido con la mayoría de alumnos en la evaluación y posterior calificación), teniendo la mayoría de alumnos un notable $(62,5 \%)$ y más alumnos en el sobresaliente $(20,83 \%)$ que en el aprobado (16,6\%). Los alumnos con peores notas se encuentran en el aprobado, debido a su actitud (pasiva, desobediente) o bien al cuaderno (no lo entregaron al día, no hacían caso a las correcciones de mejora, etc.).

\subsection{PRINCIPALES VENTAJAS ENCONTRADAS.}

Esta experiencia de EFyC ha aportado más ventajas que desventajas, tanto a al alumnado como a la profesora en prácticas que ha llevado a cabo este sistema de evaluación. Las iremos comentando a continuación:

1. Se centra al alumno en el aprendizaje: al quitar importancia a la calificación, sumas importancia al proceso de aprendizaje, dada la cercanía del alumno a los contenidos y a las situaciones de aprendizaje. Como mencionan López-Pastor, Herranz y Mínguez (2018), se supera el modelo tradicional de evaluación-calificación. Esto se ve reflejado en el diario del profesor, donde están anotados los comentarios de cada asamblea inicial y final, recordando y ampliando conocimientos sobre la sesión. Asimismo, los alumnos anotan en las autoevaluaciones sus puntos fuertes y débiles con respecto a lo que se trabaja en cada sesión (valorando el progreso personal). Pero donde mejor se ve que el alumno está centrado en el aprendizaje es en su cuaderno. 
En este instrumento se ven pruebas evidentes de que lo que ha aprendido le ha quedado claro, lo entiende y reflexiona sobre ello.

2. Feedback continuo y cercanía con los alumnos: este sistema de EFyC te permite dar un feedback más continuo e individualizado a todos los alumnos y favorecer el proceso de enseñanza-aprendizaje de los alumnos. Esta comunicación continua, ya sea oral o a través del cuaderno, hace que los alumnos te vean como un referente positivo; es decir, que te vean como un profesor que quiere ayudarles a aprender y a mejorar. Esto se puede ver claramente en las observaciones de las autoevaluaciones, comentando lo agradecidos que están con las ayudas y explicaciones que se les dan, o lo que les gusta la UD porque se ven que son capaces de mejorar.

3. Conocimiento más individualizado de los alumnos: gracias a los instrumentos de las tablas de autoevaluación, las coevaluaciones, la FSG y al diario del profesor. Como profesor estás continuamente observando y registrando aspectos concretos de cada alumno. Cuanta más información tengamos de cada alumno, más rica y objetiva será la evaluación.

4. Transparencia del profesor ante los alumnos: este sistema de EFyC parte de una explicación inicial del profesor a los alumnos sobre cómo se va a trabajar, qué van a tener que hacer los alumnos, qué hace el profesor y se muestran los instrumentos de evaluación que se van a utilizar. Sobre todo, vemos esta transparencia en la escala descriptiva (tabla 4) donde el profesor y los alumnos leen los criterios que se van a tener en cuenta durante la UD para evaluar el aprendizaje de los alumnos.

5. Se quita importancia a la calificación (nota numérica): gracias a la aplicación de este sistema de evaluación, evitamos el trabajo de modelos de educación tradicionales, los cuales son meramente transmisivos y unidireccionales, y van ligados habitualmente a sistemas de evaluación sumativos y finales (López-Pastor, Monjas, Gómez, et al. 2006). En este caso, todo el proceso de evaluación y de realización de la UD no he hablado de la nota numérica que se pone al final de la UD. Únicamente se habla de calificación con el alumnado en la explicación inicial del proceso de evaluación y, al final, en el paso a la calificación (tabla 1), donde se convierte en una calificación, en un número, la valoración realizada en la escala descriptiva (figura 3). Ni en la entrevista ni en las autoevaluaciones, ningún alumno ha mencionado "qué nota van a tener", lo cual ayuda a que se centren en el proceso de aprendizaje y no en el resultado (calificación final).

\subsection{PRINCIPALES INCONVENIENTES ENCONTRADOS $Y$ POSIBLES SOLUCIONES DE MEJORA.}

En la figura 3 presentamos los inconvenientes encontrados durante la aplicación del sistema de EFyC junto con una propuesta de soluciones de mejora hacia cada inconveniente. 


\begin{tabular}{|c|c|}
\hline $\begin{array}{l}\text { Principales inconvenientes } \\
\text { encontrados }\end{array}$ & Posibles soluciones de mejora \\
\hline $\begin{array}{l}\text { Mucho tiempo invertido en } \\
\text { realizar los instrumentos de } \\
\text { evaluación (sobre todo las } \\
\text { autoevaluaciones de cada } \\
\text { sesión) }\end{array}$ & $\begin{array}{l}\text { Se podría hacer una autoevaluación estándar para todas las } \\
\text { sesiones, clarificando en el apartado de "observaciones" } \\
\text { aspectos concretos dé cada sesión, con el fin de distinguir las } \\
\text { autoevaluaciones entre sí. } \\
\text { Otra opción sería realizar una autoevaluación más larga al } \\
\text { finalizar la UD, englobando aspectos de cada sesión. }\end{array}$ \\
\hline $\begin{array}{l}\text { Necesidad de corregir y dar } \\
\text { feedback en los cuadernos } \\
\text { cuanto antes, para que el } \\
\text { proceso de EFyC fuera } \\
\text { inmediato y real. }\end{array}$ & $\begin{array}{l}\text { Poner como norma que los alumnos deben llevar el cuaderno } \\
\text { de Educación Física a clase todas las clases de EF. El maestro } \\
\text { cada día recoge 5-8, aleatoriamente, o los que él diga. Revisa } \\
\text { el cuaderno lo antes posible y se lo devuelve corregido y con } \\
\text { feedback en la siguiente sesión. Si le señala que debe hacer } \\
\text { cambios, deben estar hechos la siguiente vez que se recoja el } \\
\text { cuaderno (aplica las correcciones para las siguientes sesiones, } \\
\text { no puede mejorar la que ya ha hecho). Esto permite que el } \\
\text { alumno reciba un feedback rápido, con indicaciones claras } \\
\text { sobre cómo ir mejorando. }\end{array}$ \\
\hline $\begin{array}{l}\text { Falta de sinceridad en las } \\
\text { autoevaluaciones (al } \\
\text { comienzo de la UD) }\end{array}$ & $\begin{array}{l}\text { Explicar la importancia de ser sinceros en las evaluaciones } \\
\text { debido a que hay un sistema de control posterior. Hacer ver a } \\
\text { los alumnos que lo que anoten en las autoevaluaciones se } \\
\text { contrasta con las anotaciones en la FSG y en el diario del } \\
\text { profesor. La clave es que ellos mismos se acostumbren a este } \\
\text { instrumento. }\end{array}$ \\
\hline $\begin{array}{l}\text { Resistencia a completar las } \\
\text { fichas de autoevaluación. } \\
\text { Como era una por sesión, } \\
\text { les parecía muy pesado y } \\
\text { monótono. }\end{array}$ & $\begin{array}{l}\text { O bien realizar una autoevaluación al final de cada semana, o al } \\
\text { final de la UD, o bien darles más tiempo para completarla sin } \\
\text { quitarles tiempo motriz (ya en clase, por ejemplo). Pienso que la } \\
\text { resistencia que muestran por rellenar este instrumento de } \\
\text { evaluación es porque les quitamos tiempo motriz para hacer } \\
\text { algo que no es nada llamativo para ellos. }\end{array}$ \\
\hline $\begin{array}{l}\text { Entrevistas muy extensas. } \\
\text { Los diálogos de los alumnos } \\
\text { se alargaron mucho y las } \\
\text { entrevistas ocuparon cerca } \\
\text { de tres clases de EF. }\end{array}$ & $\begin{array}{l}\text { Acortar mucho más el tiempo de entrevista oral con cada } \\
\text { alumno. No debemos dedicar más de una clase a realizar la } \\
\text { calificación dialogada con todo el grupo. Sintetizar la } \\
\text { información y no insistir en la conversación si el alumno y el } \\
\text { profesor están de acuerdo en los criterios. }\end{array}$ \\
\hline
\end{tabular}

Figura 3. Principales inconvenientes encontrados y posibles soluciones para cada uno de ellos.

\section{CONCLUSIONES}

Los resultados encontrados muestran que el sistema de EFyC utilizado tiene varias ventajas, pero también aspectos a mejorar.

En cuanto a las ventajas, aporta un feedback continuo, lo que le ofrece al alumnado numerosas oportunidades de mejora y aprendizaje ayuda a centrar la atención del alumnado en el proceso de aprendizaje y no el resultado final, lo que ayuda a que el alumno se motive con lo que se está trabajando. Todo ello facilita la obtención de buenos resultados académicos. El proceso de autoevaluación y autocalificación ha demostrado que los alumnos son coherentes con el aprendizaje y el trabajo realizado.

En la figura 4, se revisa el grado de consecución de los objetivos marcados para la UD, relacionada con el aprendizaje de los tipos de salud, la adquisición de 
hábitos saludables y la corrección de malos hábitos y mitos que se dan sobre la salud; y que ha sido llevada cabo con un grupo de alumnos de $5^{\circ}$ de primaria.

\begin{tabular}{|c|c|}
\hline Objetivo UD & Grado de cumplimiento \\
\hline $\begin{array}{l}\text { 1. Conocer los diferentes } \\
\text { tipos de salud de forma } \\
\text { práctica a través del } \\
\text { descubrimiento guiado. }\end{array}$ & $\begin{array}{l}\text { Durante la UD se han ido trabajando todos los tipos de salud } \\
\text { según la OMS de forma práctica. El profesor no les decía } \\
\text { exactamente lo que tenían que hacer, sino que lo iban } \\
\text { descubriendo a medida que iban trabajando. }\end{array}$ \\
\hline $\begin{array}{l}\text { 2. Formar parte del } \\
\text { proceso de aprendizaje } \\
\text { personal de la salud. }\end{array}$ & $\begin{array}{l}\text { Se ha cumplido gracias a la puesta en práctica del sistema de } \\
\text { EFyC, donde el alumno ha sido consciente de su evolución y } \\
\text { aprendizaje día a día. Los instrumentos de evaluación y el } \\
\text { feedback inmediato (profesor-alumno) han sido clave para que los } \\
\text { alumnos se sientan parte del proceso de aprendizaje. }\end{array}$ \\
\hline $\begin{array}{l}\text { 3. Reconocer los errores } \\
\text { que cometemos día a día } \\
\text { que afectan a nuestra } \\
\text { salud. }\end{array}$ & $\begin{array}{l}\text { La UD estaba enfocada de manera práctica y funcional, de modo } \\
\text { que una gran parte del aprendizaje era ver qué errores } \\
\text { cometíamos en nuestro día a día y cómo corregirlos de cara a } \\
\text { mantener una buena salud diaria. }\end{array}$ \\
\hline $\begin{array}{l}\text { 4. Llevar a cabo hábitos } \\
\text { saludables de forma } \\
\text { correcta en nuestro día a } \\
\text { día. }\end{array}$ & $\begin{array}{l}\text { Cada actividad de aprendizaje escondía unos hábitos diarios } \\
\text { mostrados como ejemplos cercanos para los alumnos. Estos } \\
\text { hábitos saludables los remarcaba el maestro en momentos de } \\
\text { reflexión-acción y en las asambleas (iniciales o finales). La UD ha } \\
\text { sido un trabajo progresivo y cíclico, ya que lo que aprendíamos un } \\
\text { día, lo trabajábamos de manera secundaria al día siguiente. }\end{array}$ \\
\hline $\begin{array}{l}\text { 5. Desarrollar un sentido } \\
\text { crítico en relación a } \\
\text { nuestra salud personal y } \\
\text { a la de los demás. }\end{array}$ & $\begin{array}{l}\text { El aprendizaje a partir del error, y de situaciones cotidianas que } \\
\text { todos los alumnos viven, hace que tomen más cercano el } \\
\text { conocimiento. Este acercamiento a lo que están aprendiendo ha } \\
\text { favorecido una buena reflexión del aprendizaje (reflejada en los } \\
\text { cuadernos y en cada asamblea), siendo críticos con lo que se ha } \\
\text { trabajado. }\end{array}$ \\
\hline
\end{tabular}

Figura 4. Grado de cumplimiento de los objetivos de la UD a partir de la experiencia de EFyC.

Uno de los aspectos clave de esta experiencia de EFyC es que ha sido planificada, puesta en práctica y evaluada por una maestra en prácticas y, por tanto, constituye su primera experiencia, sin haber terminado todavía su formación inicial. Esto puede limitar la comparación con otros estudios y experiencias llevados a cabo por profesorado en ejercicio o con mayor experiencia docente. La maestra considera que, a pesar de todo ello, se sentido muy satisfecha y cómoda durante su aplicación en un aula real. Ha sido de mucha ayuda la realización de un trabajo previo de lectura y estudio, revisando muchos trabajos publicados sobre la temática en los últimos 1015 años y planificando con tiempo su puesta en práctica, y contando con el asesoramiento de seminario de EFyC al que pertenecía y de sus tutores de trabajo fin de grado.

Por todo ello, la maestra considera que para poder llevar a cabo una experiencia de este tipo requiere dos cosas: (1) formación; (2) organización.

1. Sin formación no se puede llevar a cabo el sistema de EFyC, debido a que es un sistema muy diferente al modelo tradicional, al que todos estamos acostumbrados. Todo maestro debe formarse y llevar a la práctica lo aprendido sobre modelos de EFyC; si no es así, lo que haga puede ser erróneo, y/o no cumplir los objetivos del sistema en sí.

2. Sin organización sería inviable obtener unos resultados coherentes y prácticos con cada alumno. Se han utilizado numerosos instrumentos y se realiza una observación sistemática y continua de cada alumno, para lo que hace falta aplicar una rutina de trabajo constante. Sin esta sistematicidad y continuidad el sistema de evaluación no es real, ya que no seguiría un proceso de aprendizaje en cada alumno supervisado por el profesor.

A lo largo de esta experiencia, la maestra en prácticas ha aprendido que no por utilizar muchos instrumentos de evaluación las cosas saldrán mejor. Cuantos más 
instrumentos se elaboren, más trabajo tienen tanto el profesor como los alumnos, y, por tanto, menos abarca el profesor para supervisar y tener en cuenta cada instrumento de cada alumno. La motivación en los alumnos es clave para que el trabajo salga adelante. Por tanto es muy importante encontrar una relación equilibrada entre número de instrumentos necesarios y tiempo que implican para alumnado y profesorado.

He aprendido que este sistema de evaluación requiere de paciencia y de tiempo. Los alumnos que nunca han trabajado así lo ven algo raro y pesado (rellenar fichas de autoevaluación continuas, coevaluar, etc.); por eso los resultados que tengamos al principio no nos deben desmotivar para seguir aplicando este sistema, ya que cuanto más lo conozcan, mejores serán los resultados.

\section{REFERENCIAS}

López-Pastor, V. M. (coord.) (2006). La evaluación en Educación Física. Revisión de modelos tradicionales y planteamiento de una alternativa: la evaluación formativa y compartida. Buenos Aires: Miñó y Dávila.

López-Pastor, V. M., \& Monjas, R., \& Gómez, J., et al. (2006). La evaluación en Educación Física. Revisión de modelos tradicionales y planteamiento de una alternativa: la evaluación formativa y compartida. RETOS. Nuevas Tendencias en Educación Física, Deporte y Recreación, 10, 31-41.

López-Pastor, V. M., Herranz, M.,\& Mínguez, P. L. (2018). Evaluación formativa y compartida para una educación física crítica. En E. Lorente y D. Martos (Eds.), Educación Física y pedagogía crítica: Propuestas para la transformación personal y social (pp. 325-346). Universitat de Lleida y Universitat València.

López-Pastor, V. M., \& Pérez-Pueyo, A. (coords.) (2017). Evaluación formativa y compartida en educación: experiencias de éxito en todas las etapas educativas. León: Universidad de León. (e-book). Recuperado de https://buleria.unileon.es/handle/10612/5999 